lasted until November 2005. Gradual dose escalation up to $20 \mathrm{mg} /$ week was required during 2006 in view of RA exacerbations, nevertheless, clinical and lab RA activity persisted. Therefore tofacitinib (Jakvinus) was administered in November 2015 at $10 \mathrm{mg} /$ day, with rapid achievement of minimum RA activity (DAS28=3,0). By the end of May 2016 clinical manifestations of vasculutis emerged, and in June 2016 tofacitinib was discontinued due to increasing lab activity of the disease. Clinical examination: patient's condition is moderately grave. The thinned skin looks dry, showing multiple regenerating ulcers on the anterior leg surface and sharply painful ulcerated indurations on the right inner thigh producing serosanguinous fluid. The RA activity is equal to DAS28 $=5,72, \mathrm{HB}_{\mathrm{B}}$ $125 \mathrm{~g} / \mathrm{L}$, WBC $6,0 \times 10^{9} / \mathrm{L}$, platelets $40 \times 10^{9} / \mathrm{L}$, ESR $22 \mathrm{~mm} / \mathrm{h}, \mathrm{CRP} 42 \mathrm{mg} / \mathrm{L}, \mathrm{RF}$ $413 \mathrm{IU} / \mathrm{ml}$, antibody to cyclic citrullinated peptide (ACPA) is $195 \mathrm{U} / \mathrm{ml}$, TNF $13,9 \mathrm{pg} / \mathrm{ml}$. Wrist and distal foot radiographs show moderate extended osteoporosis, narrowing of some joint spaces, rare bone and articular surface erosions, subluxation of some joints. Histopathology of skin and subcutaneous fat biopsy samples is presented on figure 1a,1b. Histopathology data suggests the presence of cutaneous and subcutaneous fat productive and destructive vasculitis and acute, predominantly lobular, panniculitis; such structural changes are usually present in cutaneous polyarteritis nodosa and other systemic vasculitis. The casual relationship between this ADR and tofacitinib therapy was assessed as probable using Naranjo scale.

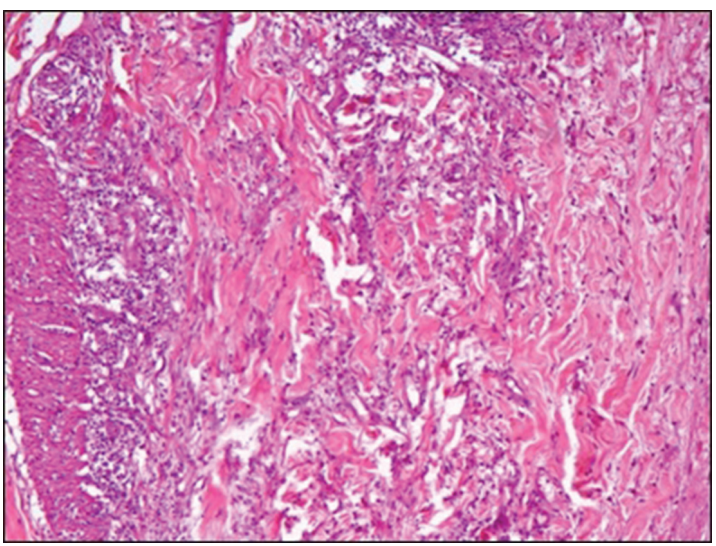

Abstract AB0413 - Figure 1

Conclusions: development of vasculitis (polyarteritis nodosa) in RA patient can be caused by tofacitinib.

Disclosure of Interest: None declared

DOI: 10.1136/annrheumdis-2018-eular.4192

\section{AB0414 RELATIONSHIP BETWEEN SERUM OXYTOCIN LEVELS AND DEPRESSION IN PATIENTS WITH RHEUMATOID ARTHRITIS}

Y. Miwa ${ }^{1}$, T. Kasama ${ }^{1}$, K. Sanada $2 .{ }^{1}$ Division Of Rheumatology, Department Of Medicine; ${ }^{2}$ Department of Psychiatry, SHOWA UNIVERSITY SCHOOL OF MEDICINE, Tokyo, Japan

Background: Oxytocin (OXT), also referred to as a happy hormone, has been associated with various psychiatric disorders, including depression. However, the relationship between depression and rheumatoid arthritis (RA) has not yet been clarified.

Objectives: The objective of this study was to investigate the relationship between serum oxytocin levels and disease activity, depressive state, activity of daily life (ADL), and quality of life (QOL) in RA.

Methods: This study included 119 RA patients. We measured the following variables: baseline characteristics including age, sex, disease duration, smoking history, body mass index, prednisolone dose, and methotrexate (MTX) dose, serum levels of rheumatoid factor, matrix metalloproteinase-3, erythrocyte sedimentation rate (ESR) and C-reactive protein (CRP) level. The disease activity of RA was assessed using the Simplified Disease Activity Index (SDAI); depression was assessed using the Hamilton Depression Rating Scale (HAM-D); ADL were assessed using the Health Assessment Questionnaire; and QOL was assessed using the 36-Item Short Form Health Survey (SF-36). Serum OXT levels were determined by enzyme-linked immunosorbent assay. The subjects were divided into two groups according to the higher or lower of the serum OXT levels, and a retrospective study was performed.

Results: The serum OXT levels were correlated with the disease duration $(r=0.443, p=0.000)$. Weak correlations of MTX dose $(r=0.208, p=0.030)$, swollen joint count (SJC) ( $r=-0.306, p=0.001)$, ESR $(r=-0.245, p=0.010), \operatorname{CRP}(r=-0.283$, $\mathrm{p}=0.003)$ and the mental component summary (MCS) score $(r=-0.196, p=0.041)$ of SF-36. The binominal logistic analyses findings were as follows: younger age $(\mathrm{p}=0.0209$, odd ratio: $0.96,95 \% \mathrm{Cl}: 0.94-0.99)$ and longer disease duration ( $p=0.0053$, odd ratio: $1.08,95 \% \mathrm{Cl}: 1.02-1.13$ ). Other items did not correlate to the serum OXT levels included with the HAM-D score.

Conclusions: The serum oxytocin levels were correlated with longer disease duration and younger age.

Acknowledgements: Cooperation on data collection: All Showa University in Rheumatoid Arthritis (ASHURA) group; Nobuyuki Yajima, Takeo Isozaki, Kuninobu Wakabayashi, Ryo Takahashi, Ryo Yanai, Hidekazu Furuya, Takahiro Tokunaga, Sakiko Isojima, Nao Oguro, Yoko Miura, Sho Ishii, Shinya Seki, Mayu Saito, Shinichiro Nishimi, Airi Nishimi, Yuzo Ikari, Mika Hatano, Tomoki Hayashi, Masahiro Hosonuma, Yoichi Toyoshima, Katsunori Inagaki and Kosuke Sakurai Disclosure of Interest: Y. Miwa Grant/research support from: Astellas Pharma Inc., Mitsubishi Tanabe Pharma Corporation, AbbVie CK, Pfizer Japan Inc., Chugai Pharmaceutical Co., Ltd., Eizai Co., Ltd, Asahi Kasei Pharm Co., Ltd, YL biologics Ltd., Japan Blood Products Organisation.. Ono Pharmaceutical Co., Ltd., Nippon Kayaku Co., Ltd., and Teijin Pharma Ltd., T. Kasama Grant/research support from: Astellas Pharma Inc., Mitsubishi Tanabe Pharma Corporation, AbbVie CK, Pfizer Japan Inc., Chugai Pharmaceutical Co., Ltd., Eizai Co., Ltd, Asah Kasei Pharm Co., Ltd, YL biologics Ltd., Japan Blood Products Organisation, Ono Pharmaceutical Co., Ltd., Nippon Kayaku Co., Ltd., and Teijin Pharma Ltd., K. Sanada: None declared

DOI: 10.1136/annrheumdis-2018-eular.3832

\section{Rheumatoid arthritis - biological DMARDs}

\section{AB0415 DISEASE FLARES AMONG EARLY RHEUMATOID ARTHRITIS PATIENTS TREATED WITH CONTINUED METHOTREXATE EITHER ALONE OR IN COMBINATION WITH ADALIMUMAB}

A. Kavanaugh ${ }^{1}$, R. van Vollenhoven ${ }^{2}$, P. Sunkureddi ${ }^{3}$, Y. Zhang ${ }^{4}$, J. Suboticki ${ }^{4}$, J. Smolen ${ }^{5}{ }^{1}$ Division of Rheumatology, Allergy, and Immunology, University of California San Diego, La Jolla, USA; ${ }^{2}$ Amsterdam Rheumatology and Immunology Center ARC, Amsterdam, Netherlands; ${ }^{3}$ University of Texas Medical Branch, Galveston; ${ }^{4}$ AbbVie Inc., North Chicago, USA; ${ }^{5}$ Division of Rheumatology, Department of Medicine, Medical University of Vienna and Hietzing Hospital, Vienna, Austria

Background: Some rheumatoid arthritis (RA) patients (pts) may experience flares in their disease even after reaching stable low disease activity (sLDA), but the consequences of even temporary elevations in disease activity are poorly understood.

Objectives: The purpose of this analysis was to explore the rates of flares after reaching sLDA in pts treated to target with either methotrexate (MTX) monotherapy or adalimumab combination therapy (ADA+MTX).

Methods: This post hoc analysis included pts from the randomised, double-blind, double-period OPTIMA ${ }^{1}$ trial achieving sLDA [DAS28(CRP) $<3.2$ at weeks (wks) 22 and 26] at the end of period 1 (P1). In P1, pts were randomised to receive ADA +MTX or placebo (PBO) +MTX for 26 wks. Pts on ADA +MTX achieving sLDA were randomised to receive PBO +MTX (ADA Withdrawal) or continue on ADA +MTX (ADA Continuation) for an additional 52 wks in period 2 (P2). Pts who achieved sLDA on PBO +MTX in P1 continued their treatment in P2 (MTX Continuation). Pts achieving sLDA in each treatment group were categorised based on whether they experienced a flare [change in DAS28(CRP) $\geq 0.6$ at consecutive visits and DAS28(CRP) $>3.2$ ]; the proportion of pts experiencing flares and time to flare were assessed. For each group, mean change from wk 26 to wk 78 in disease activity [DAS28(CRP)], functional (HAQ-DI) and structural (mTSS) measures were analysed.

Results: In pts achieving sLDA at the end of P1, flare rates in P2 differed based on initial treatment assignment (ADA Continuation: 11.7\% [11/94]; MTX Continuation: $22.4 \%$ [22/98]). Interestingly, flare rates in pts randomised to withdraw ADA in P2 (ADA Withdrawal: 25\% [22/88]) were numerically similar to the MTX Continuation group. The mean time to flare was 193, 191, and 177 days in the ADA Withdrawal, ADA Continuation, and MTX Continuation groups, respectively. During P2, the mean DAS28(CRP) scores were predictably higher in pts who flared compared with those who did not across treatment groups. In pts experiencing flares in P2, disease activity and functional measures worsened from wk 26 to wk 78 as compared with pts without flares (table 1). Of the individual DAS28(CRP) components, pt global assessment of disease activity (PtGA) showed the greatest worsening. There were small differences in radiographic progression between pts experiencing flares compared with pts without flares. 